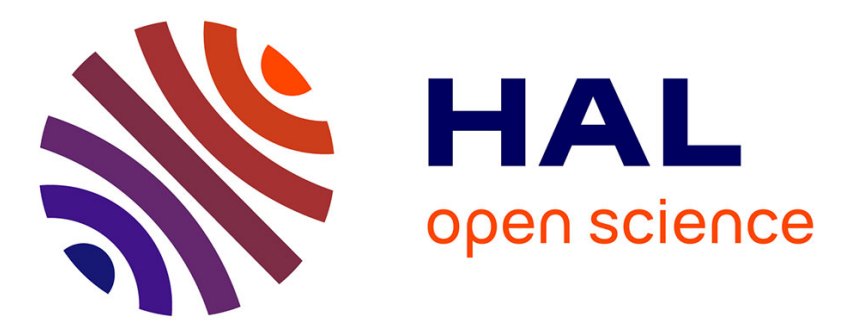

\title{
Stability Analysis and Bandwidth Estimation of Free- Carrier Driven Kerr Frequency-Comb
}

Raktim Haldar, Shailendra K Varshney, Partha Mondal, Vishwatosh Mishra

\section{To cite this version:}

Raktim Haldar, Shailendra K Varshney, Partha Mondal, Vishwatosh Mishra. Stability Analysis and Bandwidth Estimation of Free- Carrier Driven Kerr Frequency-Comb. CLEO Pacific Rim , Jul 2017, Singapour, Singapore. hal-01631398

\section{HAL Id: hal-01631398 https://hal.science/hal-01631398}

Submitted on 9 Nov 2017

HAL is a multi-disciplinary open access archive for the deposit and dissemination of scientific research documents, whether they are published or not. The documents may come from teaching and research institutions in France or abroad, or from public or private research centers.
L'archive ouverte pluridisciplinaire HAL, est destinée au dépôt et à la diffusion de documents scientifiques de niveau recherche, publiés ou non, émanant des établissements d'enseignement et de recherche français ou étrangers, des laboratoires publics ou privés. 


\section{Stability Analysis and Bandwidth Estimation of Free- Carrier Driven Kerr Frequency-Comb}

\author{
Raktim Haldar*, Shailendra K. Varshney \\ Department of E\&ECE \\ Indian Institute of Technology, Kharagpur \\ Kharagpur-721302, West Bengal, India \\ raktim@ece.iitkgp.ernet.in
}

\author{
Partha Mondal ${ }^{\dagger}$, Vishwatosh Mishra \\ Department of Physics \\ Indian Institute of Technology, Kharagpur \\ Kharagpur-721302, West Bengal, India \\ †Parthaphy1987@gmail.com
}

\begin{abstract}
Free-carrier dispersion-led nonlinear cavity detuning is observed through the reverse Kerr-tilt of the resonant-peaks of steady-state solutions of Lugiato-Lefever equation. Effects of nonlinear losses on the gain-spectrum and the gain-bandwidth of modulation-instability are also studied.
\end{abstract}

Keywords-frequency comb; microring resonators; bandwidth; modulation in-stability;nonlinear optics; Lugiato-Lefever Equation.

\section{INTRODUCTION}

Optical frequency-comb is a set of equidistant and coherent frequency lines in the infrared, visible or ultraviolet regions, which can be used in precision measurement, spectroscopy, sensing, WDM-communication or as an optical ruler [1, 2]. Silicon based platforms are preferred over other materials for the generation of on-chip optical frequency combs [2] due to several advantages of silicon such as tight optical confinement, high Kerr-coefficient, low-cost etc. Although, octave-spanning frequency combs have already been demonstrated both experimentally and numerically in $\mathrm{Si}_{3} \mathrm{~N}_{4}$ and $\mathrm{Si}$ microring resonators (MRRs) [2-4], a more realistic theoretical study on comb dynamics for Si-MRRs becomes indispensable where all the nonlinear losses and higher order dispersion terms are being considered. Nonlinear losses include multi-photon absorptions (two photon absorption (TPA/2PA), 3PA, 4PA etc.), free-carrier absorption (FCA) and free-carrier dispersion (FCD). Generalized meanfield Lugiato-Lefever equation (LLE) can be used to model the Kerr frequency comb in a high- $Q$, high-finesse optical cavity where the dispersion, nonlinear phase accumulation over a round-trip and pump detuning are low [1]. It is known that temporal cavity soliton (CS) generates Cherenkov radiation/dispersive-wave in presence of higher-order dispersions which leads octave-spanning frequency comb. Effects of Raman scattering and self-steepening on frequency comb are also investigated [2, 3]. Recently, numerical studies have shown that FCA-induced FCD causes nonlinear cavity detuning which in turn helps to generate optical frequencycomb even in absence of linear-detuning of the CW-pump [2, 3], however, a detailed theoretical study on the stability analysis of free-carrier driven Kerr-comb is still scarce. In this work, for the first time to the best of our knowledge, we find the steady state homogeneous solutions of free-carrier driven generalized mean-field LLE and report negative Kerr-tilt as a consequence of FCA-FCD assisted nonlinear cavity detuning.
We generalize the existing formulations to study the modulation instability (MI) gain and bandwidth in presence of higher-order dispersions, TPA, 3PA, 4PA, FCA and FCD.

\section{THEORETICAL STUDIES}

\section{A. Normalization of LLE}

At first we normalize the LLE and the rate-equation including multi-photon absorptions, FCA and FCD terms [2] in convenient forms, as given by equations (1) and (2), respectively. The steady-state homogeneous solutions of such equations are discussed later.

$$
\begin{array}{r}
\frac{\partial u}{\partial \xi}=-(1+j \Delta) u-\frac{1}{2}(1+j K) \phi_{c} u-j \frac{s}{2} \frac{\partial^{2} u}{\partial \tau^{2}}+\sum_{k \geq 3} \frac{d_{k}}{k !} \frac{\partial^{k} u}{\partial \tau^{k}} \\
+j|u|^{2} u-\frac{Q_{2}}{2}|u|^{2} u-\frac{Q_{3}}{3}|u|^{4} u-\frac{Q_{4}}{4}|u|^{6} u+S \\
\frac{\partial \phi_{c}}{\partial \tau}=\theta_{c_{2}}|u|^{4}+\theta_{c_{3}}|u|^{6}+\theta_{c_{4}}|u|^{8}-\frac{\phi_{c}}{\tau_{c}} \\
X=\left(1+\Delta^{2}\right) Y+\left(Q_{2}-2 \Delta\right) Y^{2}+\left\{1+\frac{Q_{2}^{2}}{4}+C_{2}(1+\Delta K)\right\} Y^{3} \\
+C_{2}\left(\frac{Q_{2}}{2}-K\right) Y^{4}+\frac{C_{2}^{2}}{4}\left(1+K^{2}\right) Y^{5} \\
X=\left(1+\Delta^{2}\right) Y-2 \Delta Y^{2}+\left(1+\frac{2}{3} Q_{3}\right) Y^{3}+C_{3}(1+\Delta K) Y^{4} \\
+\left(\frac{Q_{3}^{2}}{4}-K C_{3}\right) Y^{5}+\frac{C_{3} Q_{3}}{3} Y^{6}+\frac{C_{3}^{2}}{4}\left(1+K^{2}\right) Y^{7} \\
X=\left(1+\Delta^{2}\right) Y-2 \Delta Y^{2}+Y^{3}+\frac{Q_{4}}{2} Y^{4}+C_{4}(1+\Delta K) Y^{5} \\
-K C_{4} Y^{6}+\frac{Q_{4}^{2}}{16} Y^{7}+\frac{C_{4} Q_{4}}{4} Y^{8}+\frac{C_{4}^{2}}{4}\left(1+K^{2}\right) Y^{9} \\
X=\left[\left(1+\Delta^{2}\right) Y-2 \Delta Y^{2}+Y^{3}\right]
\end{array}
$$

\section{B. Steady-State Homogeneous Solutions}

We find the steady-state $(\partial u / \partial \tau=0)$, stationary $(\partial u / \partial \xi=0)$ homogeneous solutions of (1) for Si-MRRs in presence of TPA (telecommunication and NIR $<\sim 2.2 \mu \mathrm{m}$ ) which satisfies the quintic polynomial of output intensity, $Y\left(=|E|^{2}\right)[1]$ as given in (3). We show, septic and nonic polynomials of $Y$ as given in equations (4) and (5) express the steady-state solutions of (1) in presence of only 3PA (short wavelength IR) and 4PA (mid-IR), respectively. Note that all these three equations are 
reduced to the well-known cubic polynomial of $Y(6)$ if the nonlinear losses are neglected [1]. $C_{\mathrm{j}}$ is defined as $\left(\theta_{\mathrm{cj}} \cdot \tau_{\mathrm{c}}\right)$ while $j=2,3,4$ stands for TPA, 3PA and 4PA respectively.

(a)

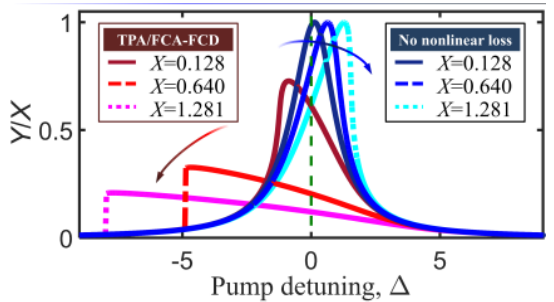

(b)

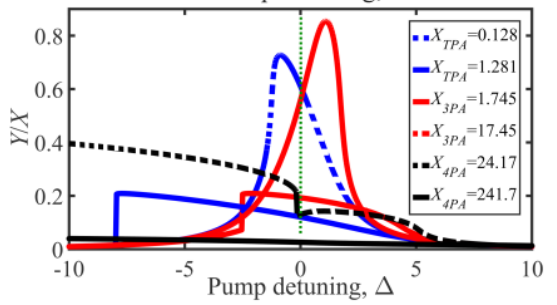

Fig. 1. (a) Kerr-tilts for the steady-state solutions of Eq. (1) in the absence of nonlinear losses (blue curves) and in presence of TPA (red-curves). (b) Kerrtilts in presence of TPA, 3PA, 4PA, FCA and FCD.

\section{Observation of Negative Kerr-tilt}

The dynamic behaviour of frequency comb can partially be understood through Kerr-tilt and bi-stability curve as the CS solutions of LLE results from the coexistence of patterned and CW solutions [1]. From Figs. 1 (a) and 1(b), it is evident that nonlinear losses introduce cavity detuning which causes reverse Kerr-tilts. We observe that the nonlinear cavity detuning introduced only by 3PA at a very low pump power does not play any vital role in comb dynamics. However, through reverse Kerr-tilt (shown by red-arrow in Fig. 1 (a)) we show that nonlinear losses especially TPA, FCA and FCD play crucial roles in determining the parameter spaces for MI, chaos formation and stable frequency-comb generations. Parameters required for the simulations are given in [2].

\section{Modulation-Instability}

Next we proceed for the stability analysis of the stationary CW solutions $\left(u_{0}\right.$ and $\left.\phi_{0}\right)$ of free-carrier driven LLE. The evolution of normalized carrier density and signal amplitude due to the spatio-temporal perturbations can be written as Eqs. (7) and (8), respectively while TPA, FCA-FCD are present.

$$
\begin{aligned}
& \phi_{c}(\xi, \tau)=\phi_{0}(\xi)+\phi_{+}(\xi) e^{i \Omega \tau}+\phi_{-}(\xi) e^{-j \Omega \tau} \\
& u(\xi, \tau)=u_{0}(\xi)+u_{+}(\xi) e^{i \Omega \tau}+u_{-}(\xi) e^{-j \Omega \tau}
\end{aligned}
$$

Thus, the stationary solutions $\left(\phi_{0}, u_{0}\right)$ and $\phi_{+}$can be given as:

$$
\begin{gathered}
\phi_{0}=\theta_{c_{2}} \tau_{c}\left|u_{0}\right|^{4}=C_{2}\left|u_{0}\right|^{4} \\
\frac{\partial u_{0}}{\partial \xi}=-(1+j \Delta) u_{0}-\frac{1}{2}(1+j K) \theta_{c_{2}} \tau_{c}\left|u_{0}\right|^{4} u_{0}+\left(j-\frac{Q_{2}}{2}\right)\left|u_{0}\right|^{2} u_{0}+S \\
\phi_{+}=\frac{2 \theta_{c_{2}} \tau_{c}\left|u_{0}\right|^{2}\left[u_{0}^{*} u_{+}+u_{0} u_{-}^{*}\right]}{\left(1+j \Omega \tau_{c}\right)}=\phi_{-}^{*}
\end{gathered}
$$

We assume, $u_{+}=C \cdot \exp (\lambda . \xi)$. Finally, the MI-gain, $\lambda$ can be found by solving the quadratic equation of $\lambda(12)$,

$$
\left(\frac{R^{2}+I^{2}}{\alpha^{2}+\beta^{2}}-Y^{2}\right)=0
$$

Here, LLE is truncated up to the 3rd order dispersions and,

$$
\begin{gathered}
R(\lambda)=-\left[(1+\lambda)+\frac{C_{2}\left(1+K \Omega \tau_{c}\right)}{1+\Omega^{2} \tau_{c}^{2}} Y^{2}+\frac{C_{2}}{2} Y^{2}+Y Q_{2}\right] \\
I(\lambda)=-\left[\Delta+\frac{C_{2}\left(K-\Omega \tau_{c}\right)}{\left(1+\Omega^{2} \tau_{c}^{2}\right)} Y^{2}+\frac{K C_{2}}{2} Y^{2}-\frac{s}{2} \Omega^{2}+\frac{\Omega^{3}}{6} d_{3}-2 Y\right] \\
\alpha=\left[C_{2} \frac{\left(1+K \Omega \tau_{c}\right)}{\left(1+\Omega^{2} \tau_{c}^{2}\right)} Y+\frac{Q_{2}}{2}\right] \\
\beta=\left[C_{2} \frac{\left(K-\Omega \tau_{c}\right)}{\left(1+\Omega^{2} \tau_{c}^{2}\right)} Y-1\right]
\end{gathered}
$$

Note that, in absence of nonlinear losses, $\lambda$ reduces to the well-known expression (17) for MI-gain:

$$
\lambda_{ \pm}=-1 \pm \sqrt{4 Y\left(\Delta-\frac{s \Omega^{2}}{2}\right)-\left(\Delta-\frac{s \Omega^{2}}{2}\right)^{2}-3 Y^{2}}
$$

Figs. 2(a) and (b) show that the presence of third-order dispersion, $d_{3}(=0.1)$ can enhance the gain-bandwidth whereas, the MI-gain for Si-waveguide, in presence of TPA, FCA and FCD is insufficient to create any sidebands.

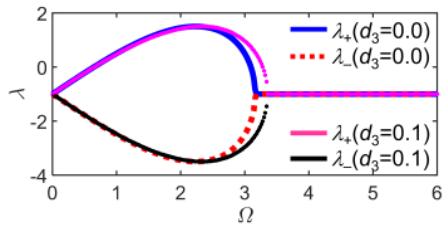

(a)

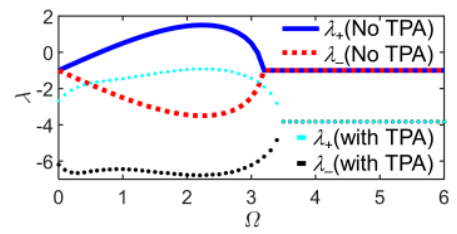

(b)
Fig. 2 (a) Effect of $3^{\text {rd }}$ order dispersion and (b) TPA, FCA-FCD on $\lambda . s=1, Y=2.5, \Delta=7.5, Q_{2}=1, K=0.05, C_{2}=0.1, \theta_{\mathrm{c} 2}=0.05$ taken. For $\mathrm{Si}$ at wavelength $<2.2 \mu \mathrm{m}$ TPA is even higher to yield $\lambda \sim-100$ for the entire $\Omega$ range [0-6] shown here.

\section{NUMERICAL RESUltS}

Our theoretical predictions are confirmed numerically by solving the LLE (1) and the rate-equation (2) through split-step Fourier method (Fig. 3). It is observed that TPA (with FCAFCD) inhibits the comb formation whereas 3PA can reduce the comb-bandwidth significantly.

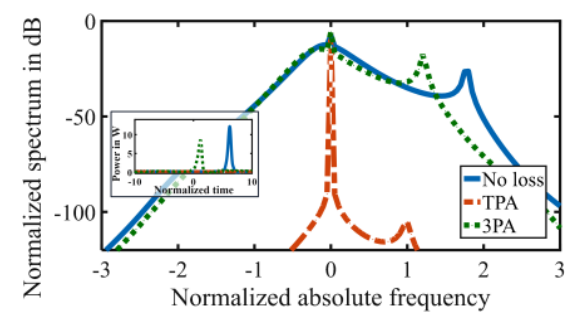

Fig. 3 Numerical simulations confirm that a different dynamics is being played for different parameter spaces of $\Delta$ and $X$ in presence of nonlinear losses. Inset shows the final pulses in time domain. $\theta_{\mathrm{c} 2}=0.0005, Q_{2}=0.93, C_{2}=29.81, K_{2}=7.5$, $d_{3}=0.1$ (at $\left.\lambda_{0} \sim 1.56 \mu \mathrm{m}\right) . \theta_{\mathrm{c} 3}=6.31, Q_{3}=0.087, C_{3}=0.049, K_{3}=4.9$ (at $\left.\lambda_{0} \sim 2.4 \mu \mathrm{m}\right)$.

\section{CONCLUSION}

To conclude, nonlinear phase detuning of cavity has been observed through negative Kerr-tilt. For the first time to the best of our knowledge, we also find out the MI-gain and bandwidth for Kerr-comb in presence of nonlinear losses.

\section{References}

[1] S. Coen et. al, Opt Lett. 38, 1790-1792 (2013).

[2] R. K. W. Lau et al., Opt. Lett. 40, 2778-2781 (2015).

[3] T. Hansson et al., Opt. Lett. 39, 6747-6750 (2014).

[4] A. G. Griffith et al., Nat. Commun. 6, 6299 (2015). 